\title{
Tratamiento endovascular y trombólisis intraarterial en el ictus isquémico agudo
}

\author{
D. Escudero ${ }^{a, *}$, R. Molina ${ }^{a}$, L. Viña ${ }^{a}$, P. Rodríguez ${ }^{a}$, L. Marqués ${ }^{a}, E$. Fernández ${ }^{a}$,

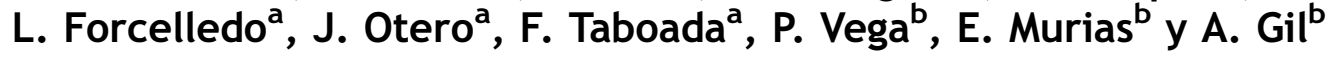

${ }^{a}$ Servicio de Medicina Intensiva, Hospital Universitario Central de Asturias, Oviedo, España

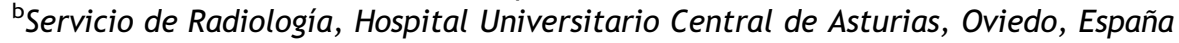

Recibido el 10 de noviembre de 2009; aceptado el 23 de enero de 2010

Disponible en Internet el 23 de marzo de 2010

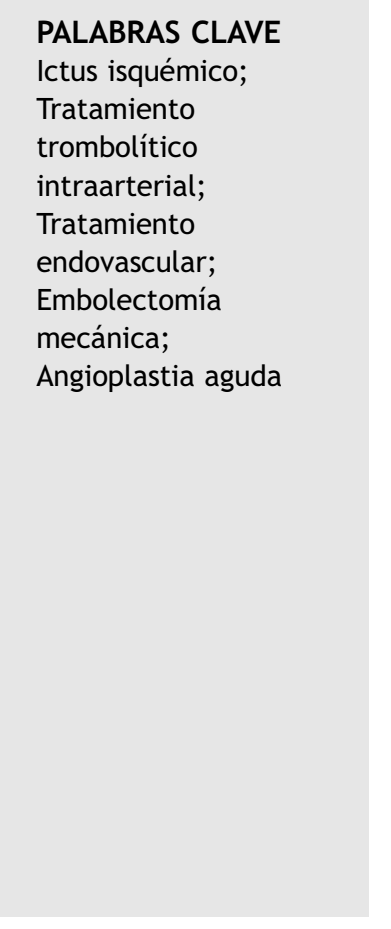

\begin{abstract}
Resumen
Objetivo: Analizar la eficacia y la seguridad de la trombólisis intraarterial y el tratamiento endovascular en pacientes con ictus isquémico agudo.

Diseño y ámbito: Estudio prospectivo observacional en una unidad de cuidados intensivos. Pacientes: Dieciséis pacientes recibieron tratamiento endovascular. Se recogieron datos epidemiológicos, localización de la oclusión arterial, tiempo desde el ictus al tratamiento, indicación del tratamiento, National Institutes of Health Stroke Scale al ingreso y al alta hospitalaria, y complicaciones y evolución funcional por escala de Rankin modificada realizada mediante una encuesta telefónica.

Resultados: Diez varones, con una edad media de 59 años (29-74) y una estancia media en la unidad de cuidados intensivos de 6 días $(1-33)$. Siete pacientes requirieron ventilación mecánica. Indicación del tratamiento: 4 casos por fracaso de la trombólisis intravenosa, 5 por oclusión de gran vaso, 2 por estar fuera de la ventana terapéutica, 3 por oclusión de la circulación posterior, uno por estar fuera de la ventana terapéutica y tener, además, una oclusión de gran vaso y uno por contraindicación para la trombólisis intravenosa. Localización de la oclusión: 3 en la circulación posterior y 13 en el territorio carotídeo y sus ramas. El fibrinolítico utilizado fue uroquinasa en dosis de 100.000 600.000 UI. Cuatro pacientes requirieron embolectomía mecánica y 10, implantación de stent. Se obtuvo recanalización completa en 11 pacientes $(69 \%)$ y parcial en $4(25 \%)$. Tres pacientes evolucionaron a muerte encefálica. Seis pacientes $(46 \%)$ tuvieron una buena recuperación (escala de Rankin modificada $\leq 2$ ). Como complicación técnica destacó un seudoaneurisma de la arteria femoral.

Conclusiones: El tratamiento intraarterial obtiene altas tasas de recanalización y buenos resultados funcionales con escasas complicaciones. Su uso estaría indicado en pacientes
\end{abstract}

\footnotetext{
*Autor para correspondencia.

Correos electrónicos: dolores.escudero@sespa.princast.es, lolaescudero@telefonica.net (D. Escudero).
} 


\section{KEYWORDS}

Ischemic stroke;

Intra-arterial

thrombolytic therapy;

Endovascular

treatment;

Mechanical

embolectomy;

Acute angioplasty

con afectación neurológica importante (National Institutes of Health Stroke Scale $\geq 10$ ) - tiempo de evolución de $3-6 \mathrm{~h}$ - y contraindicacio'n para la trombólisis intravenosa y la oclusión arterial proximal.

(c) 2009 Elsevier España, S.L. y SEMICYUC. Todos los derechos reservados.

\title{
Endovascular treatment and intra-arterial thrombolysis in acute ischemic stroke
}

\begin{abstract}
Objective: Analysis of the safety and efficacy of intra-arterial thrombolysis therapy and endovascular treatment in acute ischemic stroke.

Design and area: An observational prospective study in the Intensive Care Unit.

Patients and methods: 16 patients had endovascular treatment. Epidemiological data, arterial occlusion site, time between stroke onset and treatment, treatment indication, NIHSS scale at admission and discharge from hospital, complications and functional outcome measured by modified Rankin scale (obtained by telephone survey) were collected.

Results: Ten male patients with a mean age of 59 years $(29-74)$ were included. The mean stay in the ICU was 6 days (1-33). Seven patients required mechanical ventilation. Treatment indications were: intravenous thrombolysis failure in 4 patients, major vessel occlusion in 5 , outside of the therapeutic window in 2 , posterior circulation occlusion in 3 , outside of the therapeutic window plus major vessel occlusion in 1 and intravenous thrombolysis contraindication in 1. The occlusion site was on posterior circulation in 3 and on carotid territories and branches in 13. Thrombolytic treatment used was Urokinase at a dose of 100,000-600,000 IU. Four patients required mechanical embolectomy and 10 stent implantation. Complete recanalization was observed in 11 (69\%) and partial in 4 (25\%). Three evolved to brain death. Six patients (46\%) had a favorable outcome (modified Rankin scale score $\leq 2$ ). Technical complication was 1 femoral artery pseudoaneurysm.

Conclusions: With the intra-arterial treatment, high rates of recanalization and favorable outcome are obtained with few complications. It could be indicated in patients with severe neurological injury (NIHSS $\geq 10$ ), evolution time between $3-6 \mathrm{~h}$, intravenous thrombolysis contraindication and proximal arterial occlusion.
\end{abstract}

(c) 2009 Elsevier España, S.L. and SEMICYUC. All rights reserved.

\section{Introducción}

El 80 - $85 \%$ de los ictus son de tipo isquémico; el origen más frecuente es la oclusión embólica a partir del corazón o desde lesiones arterioescleróticas de las arterias proximales. El ictus isquémico es la tercera causa de muerte en los países desarrollados y la primera causa de discapacidad en adultos. La enfermedad provoca un efecto devastador sobre la calidad de vida del paciente $\mathrm{y}$, además, supone un altísimo coste sanitario ${ }^{1-3}$.

La oclusión arterial condiciona una disminución del flujo sanguíneo cerebral (FSC), cuyas consecuencias dependerán del nivel de flujo. Cuando el FSC es inferior a $10 \mathrm{ml} /$ $100 \mathrm{~g} / \mathrm{min}$ se produce la muerte celular y el infarto; entre la zona infartada y el parénquima cerebral con FSC normal ( $>50 \mathrm{ml} / 100 \mathrm{~g} / \mathrm{min}$ ) hay una zona hipoperfundida de extensión variable según los mecanismos de compensación hemodinámica, en la que se distinguen 2 áreas bien diferenciadas: la oligohémica, con un FSC superior a $22 \mathrm{ml} / 100 \mathrm{~g} / \mathrm{min}$ y que raramente evoluciona a tejido infartado, y la penumbra isquémica, con un FSC inferior a $22 \mathrm{ml} / 100 \mathrm{~g} / \mathrm{min}$ y que progresa a infarto cerebral si no se restablece precozmente la perfusión. El objetivo fundamental en el tratamiento del ictus es recanalizar de manera urgente la arteria obstruida y conseguir la reperfusión precoz del tejido cerebral. Este concepto se ha resumido gráficamente en el eslogan «tiempo es cerebro». Además de la recanalización de la arteria obstruida, es fundamental el tratamiento general del paciente en unidades de ictus o unidades de cuidados intensivos $(\mathrm{UCl})$ especializadas, con lo que se consigue un importante beneficio terapéutico.

El tratamiento trombolítico intravenoso con el activador tisular del plasminógeno recombinante (rt-PA) es un tratamiento de eficacia demostrada desde la publicación del ensayo clínico The National Institute of Neurological Disorders and Stroke (NINDS) ${ }^{4}$, y supuso un importante avance en el tratamiento de la enfermedad. El rt-PA se administra en una ventana terapéutica de $3 \mathrm{~h}$ desde el inicio de los síntomas, por lo que solamente se puede beneficiar un pequeño porcentaje de pacientes ${ }^{4,5}$. Estudios epidemiológicos realizados en nuestro medio han constatado que sólo la mitad de los pacientes acuden al hospital dentro de las 3 primeras horas ${ }^{6}$. Algunos trabajos han demostrado el beneficio terapéutico de la trombólisis intravenosa más allá de las 3 primeras horas, pero ampliar la ventana terapéutica requiere estudios de neuroimagen multimodales (TC de perfusión o RM de difusión/perfusión) que confirmen la presencia de áreas cerebrales de penumbra potencialmente recuperables $^{7-9}$.

Existen estudios que avalan la utilización del tratamiento trombolítico intravenoso seguido del intraarterial ${ }^{10-13}$ y que 
demuestran la eficacia, e incluso la superioridad, del tratamiento trombolítico exclusivamente intraarterial frente al intravenoso en algunos grupos de pacientes ${ }^{14-15}$. La trombólisis intraarterial con o sin extracción mecánica del trombo permite ampliar la ventana terapéutica y tratar a pacientes con más de $3 \mathrm{~h}$ de evolución, o puede considerarse una opción de rescate cuando existe contraindicacion para la trombólisis intravenosa o ésta no ha sido eficaz, hecho que ocurre frecuentemente en casos de obstrucción completa y de los grandes vasos (segmento M1 de la arteria cerebral media [ACM] o arteria carótida interna).

El objetivo del presente estudio fue analizar los resultados del tratamiento con trombólisis intraarterial y el tratamiento endovascular en pacientes con ictus isquémico agudo ingresados en una $\mathrm{UCl}$.

\section{Pacientes y métodos}

\section{Diseño y ámbito}

Estudio prospectivo de tipo observacional en un servicio de medicina intensiva de hospital universitario y centro de referencia para neurocirugía.

\section{Análisis estadístico}

Estadística descriptiva. Los datos se expresan en media \pm desviación estándar.

\section{Población estudiada}

El periodo de estudio fue de febrero de 2006 a junio de 2009. Fueron analizados 16 pacientes con ictus isquémico que recibieron tratamiento trombolítico intraarterial 0 tratamiento endovascular de forma consecutiva. Según la disponibilidad, se realizó previamente TC craneal sin contraste o angio-TC de troncos supraaórticos y TC de perfusión cerebral. Se recogieron datos epidemiológicos, localización de la oclusión, indicación de la trombólisis intraarterial, tiempo desde el inicio de los síntomas hasta el tratamiento, tipo de tratamiento, resultados arteriográficos del tratamiento endovascular, situación clínica al ingreso y al alta hospitalaria medida por la National Institutes of Health Stroke Scale (NIHSS), y complicaciones presentadas. La evolución clínica de los pacientes se realizó mediante una encuesta telefónica utilizando la escala de Rankin modificada.

\section{Protocolo de tratamiento}

El tratamiento endovascular se realizó siempre por parte de neurorradiólogos. Se consideró contraindicación absoluta la presencia de hemorragia cerebral o un descenso significativo del volumen sanguíneo cerebral en la TC de perfusión en los pacientes con más de $3 \mathrm{~h}$ de evolución. Las indicaciones del tratamiento endovascular se pueden ver en la tabla 1. A todos los pacientes se les realizó a través de la arteria femoral una arteriografía cerebral completa para diagnosticar el punto de oclusión y la colateralidad en
Tabla 1 Indicaciones del tratamiento endovascular

- Ictus isquémico agudo con NIHSS $\geq 10$

- $<3 \mathrm{~h}$ de evolución con diagnóstico por neuroimagen de las siguientes oclusiones:

a. Oclusión vascular en circulación posterior (vertebrobasilar)

b. Oclusión de gran vaso: $\mathrm{ACl}$ y trombo «en T» (carótida más segmentos proximales de la ACM y la ACA).

c. Oclusión en el segmento M1 de la ACM cuando:

- Existe contraindicación para el tratamiento trombolítico intravenoso

- La trombólisis intravenosa no ha sido eficaz

- Entre 3-6h de evolución si el territorio en penumbra es mayor del $20 \%$ del territorio isquémico en los siguientes casos:

- Oclusión en la $\mathrm{ACl}$, la bifurcación intracraneal y el segmento M1 de la ACM

Oclusión en la circulación posterior (vertebrobasilar)

ACA: arteria cerebral anterior; $\mathrm{ACl}$ : arteria carótida interna; ACM: arteria cerebral media; NIHSS: National Institutes of Health Stroke Scale.

cada caso. Los procedimientos se realizaron con sedación o anestesia general dependiendo del estado del paciente. Para la trombólisis farmacológica intraarterial se avanzó una microguía y un microcatéter hasta el punto de oclusión; posteriormente, se atravesó el trombo y se realizaron inyecciones de contraste distales a éste para valorar su extensión. Se administró uroquinasa (Vedim Fharma, Barcelona España) a un ritmo de 10.000 Ul/min. El microcatéter se retiraba progresivamente mientras se administraba el fármaco en el seno del trombo. La dosis máxima utilizada fue de 600.000 UI.

La trombólisis mecánica se realizó mediante la extracción mecánica del trombo, la angioplastia y la liberación de stent, quedando a criterio del neurorradiólogo según valoración individual de cada caso clínico, la elección y el tipo de la/s técnicas utilizadas. Generalmente, la trombólisis mecánica se iniciaba realizando varios pases con la microguía y el microcatéter para conseguir la fragmentación del trombo. Cuando no se obtenía la recanalización, se realizaba la angioplastia con un balón de baja presión (Hyperglide EV3, California, EE. UU., o Hiperform EV3) y la liberación de los stents para comprimir/aplastar el trombo contra la pared del vaso (Neuroform3 o Wingspan, Boston Scientific, Natick, EE. UU.). En 2 pacientes se utilizó como dispositivo extractor un stent autoexpandible totalmente recuperable (Solitaire EV3, California, EE. UU.).

Las oclusiones de la arteria carótida interna extracraneal se trataron mediante angioplastia (Ultra-Soft SV, Boston Scientific, Natick, EE. UU.) y stent (Acculink, Abbott Vascular, Santa Clara, EE. UU. y Wallstent Boston Scientific, Natick, EE. UU.) después de haber atravesado el punto de oclusión con una microguía (Guide Wire GT, Terumo, Tokio, Japón) y un microcatéter (SL-10, Boston Scientific, Natick, EE. UU. y Rapid Transit Cordes, Miami, EE. UU.).

Durante el procedimiento se realizó heparinización intravenosa con bolo inicial de $2.000 \mathrm{UI}$ de heparina sódica y perfusión de $1.000 \mathrm{UI} / \mathrm{h}$. Cuando se implantaba el stent se 


\begin{tabular}{|c|c|c|c|c|c|c|c|c|c|c|c|c|c|c|c|c|}
\hline Paciente $\mathrm{n} .^{\circ}$ & 1 & 2 & 3 & 4 & 5 & 6 & 7 & 8 & 9 & 10 & 11 & 12 & 13 & 14 & 15 & 16 \\
\hline Edad & 72 & 44 & 72 & 63 & 29 & 72 & 42 & 62 & 64 & 74 & 57 & 57 & 62 & 58 & 74 & 46 \\
\hline Sexo & M & v & M & v & M & v & v & M & M & M & v & v & $\mathrm{v}$ & $\mathrm{v}$ & v & v \\
\hline $\begin{array}{l}\text { Localización de la oclusión } \\
\text { arterial }\end{array}$ & $\begin{array}{l}\text { ACM D } \\
\text { segmento M1 }\end{array}$ & $\begin{array}{l}\mathrm{ACl}+ \\
\mathrm{ACM} \mathrm{I}\end{array}$ & $A B$ & $\begin{array}{l}\mathrm{ACl}+\mathrm{ACM} \\
\mathrm{I}\end{array}$ & $\begin{array}{l}\mathrm{ACl}+\mathrm{ACM} \\
\mathrm{I}\end{array}$ & $\mathrm{ACl}+\mathrm{ACM} \mathrm{D}$ & $A C M D$ & $\begin{array}{l}\text { ACM D } \\
\text { segemento M1 }\end{array}$ & $\begin{array}{l}\text { ACM I } \\
\text { segmento M2 }\end{array}$ & $\mathrm{ACl}+\mathrm{ACM} \mathrm{I}$ & $\mathrm{AB}+\mathrm{ACP} \mathrm{I}$ & $\begin{array}{l}\mathrm{ACl}+\mathrm{ACM} \\
\mathrm{I}+\mathrm{ACA} I\end{array}$ & $\begin{array}{l}\mathrm{ACl}+\mathrm{ACM} \\
\mathrm{I}+\mathrm{ACA} \mathrm{I}\end{array}$ & $\mathrm{ACl}$ & $A C I D I$ & $\mathrm{AB}+\mathrm{AV}$ \\
\hline Diagnóstico & TC, TC & TC, TC & TC & TC & $\begin{array}{l}\text { TC } \\
\text { craneal }\end{array}$ & TC & TC & TC, TCP & TC, TCP & TC craneal + & TC & $\mathrm{TC}, \mathrm{TCP}$ & TC craneal & $\mathrm{TC}$ & TC TCP & TC \\
\hline Trombólisis intravenosa & Sí & No & No & No & No & Sí & Sí & No & No & No & No & No & Sí & No & No & No \\
\hline Indicación de TIA & FTIV & OGV & $\mathrm{CP}$ & $\mathrm{FVT}+\mathrm{OGV}$ & FVT & FTIV & FTIV & FVT & CTIV & OGV & $\mathrm{CP}$ & OGV & FTIV & OGV & OGV & $\mathrm{CP}$ \\
\hline Trombolítico, unidades & UK, 240.000 & $\begin{array}{l}\text { UK, } \\
150.000\end{array}$ & $\begin{array}{l}\text { UK, } \\
500.000\end{array}$ & No & $\begin{array}{l}\text { UK, } \\
500.000\end{array}$ & UK, 200.000 & $\begin{array}{l}\text { UK, } \\
100.000\end{array}$ & No & UK, 500.000 & UK, 240.000 & UK, 250.000 & UK, 500.000 & UK, 500.000 & No & $\begin{array}{l}\text { UK, } \\
250.0000\end{array}$ & UK, 600.000 \\
\hline AA intravenosos & No & No & $\mathrm{AX}$ & $A C X$ & No & No & No & No & No & No & $A C X$ & No & No & No & No & No \\
\hline Angioplastia & Sí & No & Sí & Sí & No & No & Sí & Sí & No & No & No & Sí & Sí & Sí & Sí & Sí \\
\hline Embolectomía mecánica & No & No & Sí & Sí & Sí & No & Sí & No & No & No & No & No & No & No & No & No \\
\hline Implantación de stent & No & Sí ACl & Sí & Sí $\mathrm{ACl}$ & No & Sí ACl & No & No & No & Sí ACl & Sí & Sí ACl & No & $\begin{array}{l}\text { Sí } \\
\text { ACl }\end{array}$ & Sí ACl & Sí AV \\
\hline $\begin{array}{l}\text { Tiempo desde el ictus hasta } \\
\text { la TIA, } h\end{array}$ & 3 & 5 & 6 & 6 & 5 & $31 / 2$ & $21 / 2$ & 14 & 3 & 3 & 5 & 5 & 6 & 3 & 4 & 4 \\
\hline Recanalización & Sí & Sí & Sí & Sí & Sí & Sí & Sí & Sí & $\mathrm{RP}$ & Sí & RPAB & $\mathrm{RPACl}$ & No & Sí & Sí & RPAV AB \\
\hline NIHSS al ingreso & 17 & 20 & 27 & 20 & 20 & 15 & 12 & 15 & 9 & 24 & 20 & 20 & 25 & 12 & 25 & 25 \\
\hline NIHSS al alta del hospital & 12 & 2 & 3 & 6 & 10 & 15 & 2 & 1 & 4 & Fallecimiento & 12 & Fallecimiento & 15 & 2 & 10 & Fallecimiento \\
\hline VM, d & No & No & S 1 & No & No & Sí 1 & No & No & No & Sí 3 & Sí 15 & SI 2 & No & No & Sí 14 & Si 1 \\
\hline Días de estancia en la UCl & 5 & 2 & 2 & 4 & 6 & 2 & 1 & 1 & 1 & 3 & 33 & 4 & 8 & 1 & 18 & 1 \\
\hline Complicaciones técnicas & No & SA & No & No & No & No & No & No & No & No & No & No & No & No & No & No \\
\hline Otras complicaciones & & & & & & HICS & & & & $\begin{array}{l}\text { Infarto } \\
\text { masivo, HIC }\end{array}$ & $\begin{array}{l}\text { Hidrocefalia, drenaje } \\
\text { ventricular }\end{array}$ & $\begin{array}{l}\text { Infarto } \\
\text { masivo, HIC }\end{array}$ & & & Neumonía & $\begin{array}{l}\text { Infarto masivo, } \\
\text { HIC }\end{array}$ \\
\hline $\begin{array}{l}\text { E. Rankin (M) } \\
\text { Seguimiento }\end{array}$ & $\begin{array}{l}6 \\
\text { fallecimiento }\end{array}$ & 0 & 0 & 4 & 3 & $\begin{array}{l}6 \\
\text { fallecimiento }\end{array}$ & 0 & 0 & 2 & $\begin{array}{l}\text { Fallecimiento } \\
\mathrm{ME}\end{array}$ & 3 & $\begin{array}{l}\text { Fallecimiento } \\
\mathrm{ME}\end{array}$ & 5 & 0 & 3 & $\begin{array}{l}\text { Fallecimiento } \\
\mathrm{ME}\end{array}$ \\
\hline (meses) & 2 & 31 & 41 & 27 & 35 & 3 & 11 & 16 & 8 & & 16 & & 2 & 27 & 25 & \\
\hline
\end{tabular}

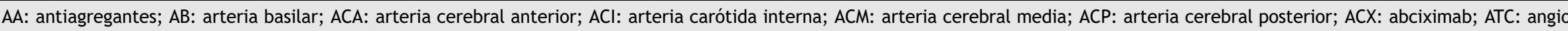

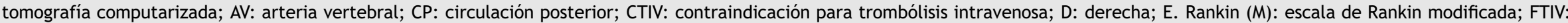

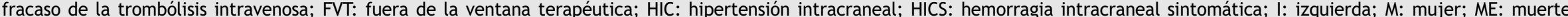

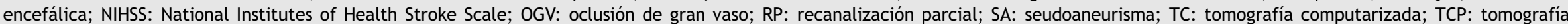
computorizada de perfusión; TIA: trombólisis intraarterial; TIV: trombólisis intravenosa; UCI: unidad de cuidados intensivos; UK: uroquinasa; V: varón; VM: ventilación mecánica. 
administraban, además, antiagregantes por sonda nasogástrica ( $300 \mathrm{mg}$ de clopidogrel y $500 \mathrm{mg}$ de AAS) o un inhibidor de la glucoproteína IIb/Illa (abciximab) intravenoso (bolo de $0,25 \mathrm{mg} / \mathrm{kg}$ seguido de perfusión intravenosa continua a $0,125 \mu \mathrm{g} / \mathrm{kg} / \mathrm{h}$ durante $12 \mathrm{~h}$ ). Tras el procedimiento endovascular, todos los pacientes fueron ingresados en la $\mathrm{UCl}$, motorizados con los parámetros habituales y un estricto control del nivel de conciencia y exploración neurológica. El tipo y el nivel de tratamiento médico dependieron de la situación clínica del paciente y se realizaron siguiendo guías de tratamiento estándares. A todos los pacientes se les realizó una TC craneal de control $24 \mathrm{~h}$ después del tratamiento trombolítico.

\section{Resultados}

Las características clínicas de los pacientes estudiados y parte de los resultados pueden verse en la tabla 2 . Se trató a 16 pacientes (10 varones y 6 mujeres), con una edad media de 59,2 $\pm 13,1$ años (rango 29-74). La estancia media en la $\mathrm{UCl}$ fue de 5,6 $\pm 8,4$ días $(1-33)$. Siete pacientes requirieron intubación y ventilación mecánica (1 15 días).

A 4 pacientes se les realizó inicialmente tratamiento con rt-PA intravenoso. La indicación del tratamiento endovascular fue la siguiente: 4 casos por fracaso de la trombólisis intravenosa, 5 por oclusión de gran vaso, 2 por estar fuera de la ventana terapéutica de $3 \mathrm{~h}, 3$ por oclusión de la circulación posterior, uno por estar fuera de la ventana terapéutica y tener, además, una oclusión proximal, y un paciente por contraindicación para la fibrinólisis intravenosa debida a un angioma cavernoso. La localización de la lesión y de las arterias ocluidas se puede ver en la tabla 2.

Cuatro pacientes requirieron embolectomía mecánica y $10(62,5 \%)$ precisaron la implantación de stent. El tiempo en horas desde el inicio de los síntomas hasta el tratamiento endovascular osciló entre 3 y $6 \mathrm{~h}$. Como excepción, el caso n. ${ }^{\circ} 9$, que ingresó con NIHSS de 15 , se recuperó de forma espontánea con NIHSS de 1. Catorce horas después, volvió a presentar clínica neurológica (hemiparesia, disartria y afectación del nervio facial con NIHSS de 15), motivo por el que se decidió tratamiento intraarterial.
Se consiguió recanalización completa en 11 pacientes $(68,7 \%)$, recanalización parcial en 4 pacientes $(25 \%)$ y recanalización nula en un paciente. Un paciente $(6,2 \%)$ presentó hemorragia intracraneal sintomática y otros 3 $(18,7 \%)$ desarrollaron infarto masivo de la ACM, gran edema cerebral, síndrome de hipertensión endocraneal refractario al tratamiento y evolución a muerte encefálica. Otros 2 pacientes fallecieron a los 2 y 3 meses del tratamiento endovascular, uno de ellos por un infarto agudo de miocardio. No hubo complicaciones durante el procedimiento endovascular y como complicación técnica hay que destacar un seudoaneurisma de la arteria femoral que requirió tratamiento quirúrgico.

La evolución funcional medida por la escala de Rankin modificada y realizada mediante una encuesta telefónica está recogida en la tabla 2 . De los pacientes a los que se les dio el alta, 6 (46\%) eran independientes para las actividades de la vida diaria y tenían escala de Rankin modificada inferior o igual 2.

En las figuras 1 y 2 se pueden ver las diferentes imágenes antes, durante y después del tratamiento endovascular del caso n. ${ }^{\circ}$ 3: mujer de 72 años con ictus isquémico agudo por obstrucción de la arteria basilar, grave y rápido deterioro del nivel de conciencia, y tetraparesia que precisó intubación orotraqueal y ventilación mecánica. Se consiguió recanalización completa tras el tratamiento con uroquinasa intraarterial e implantación del stent. El control realizado en la $\mathrm{UCl}$ mediante sonografía Doppler transcraneal mostró la recanalización de la circulación posterior con permeablidad completa de la arteria basilar. Se extubó a la paciente a las $24 \mathrm{~h}$ y dada de alta al Servicio de Neurología con NIHSS 3. Otros casos clínicos se pueden ver en las figuras 3,4 y 5 .

\section{Discusión}

En pacientes con oclusión arterial muy proximal, de origen aterotrombótico y con NIHSS elevado, la respuesta al tratamiento con rt-PA intravenoso es baja. La eficacia y la seguridad del tratamiento dependen de su precocidad y disminuyen progresivamente según el aumento del tiempo
A

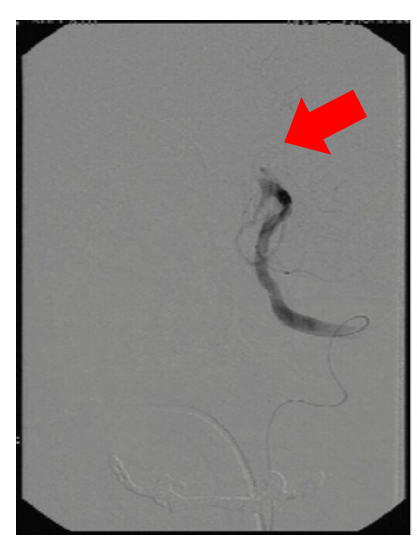

B

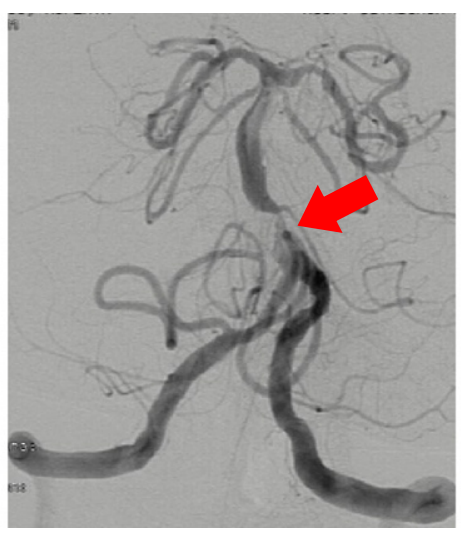

C

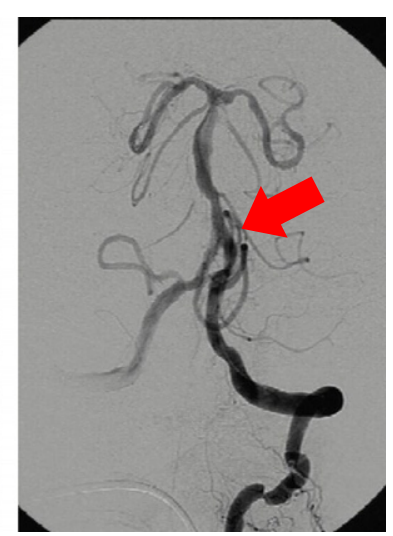

Figura 1 Paciente con ictus isquémico agudo de la circulación posterior. A) Arteriografía cerebral que muestra obstrucción de la arteria basilar. B) Recanalización tras la trombólisis con uroquinasa intraarterial y estenosis grave por una placa de ateroma. C) Control final de la arteria basilar, ya totalmente permeable tras la colocación del stent. 
A

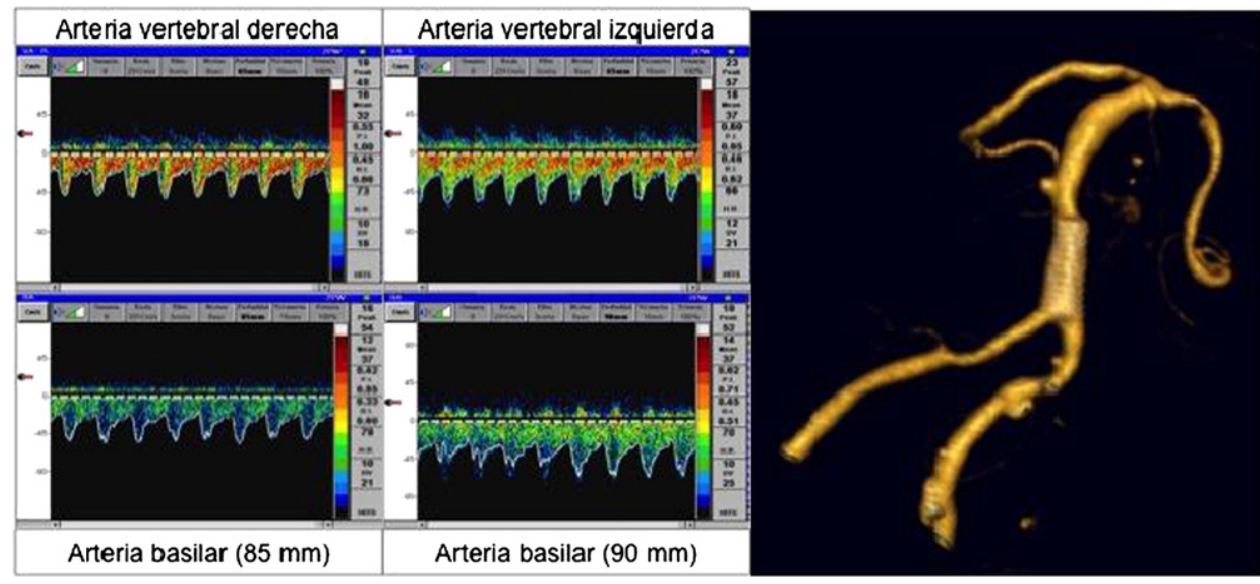

Figura 2 Evolución de la paciente de la figura 1. A) Control con sonografía Doppler transcraneal posterior al tratamiento endovascular; arterias vertebrales y arteria basilar permeables con velocidad media e índices de pulsatilidad normales. B) Reconstrucción tridimensional de la angio-TC que muestra normalidad del flujo en la arteria basilar.
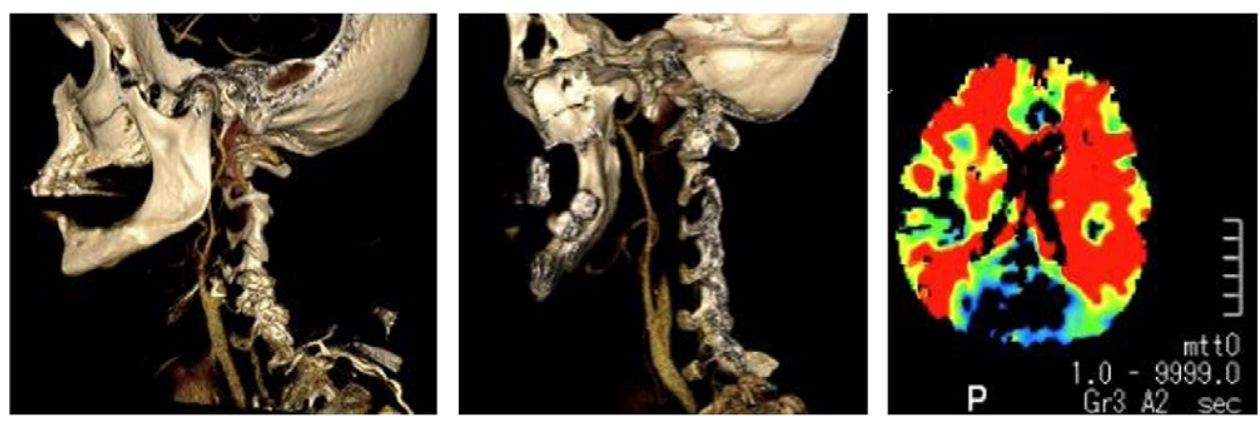

Figura 3 Angio-TC con obstrucción crónica de la arteria carótida interna izquierda y aguda en la arteria carótida interna derecha. TC de perfusión con alteración del tiempo de tránsito medio en ambos hemisferios cerebrales.

de evolución ${ }^{4,16,17}$. Utilizando técnicas de imagen multimodal se ha visto que hasta en el $80 \%$ de los casos con $3-6 \mathrm{~h}$ de evolución existe un área de tejido en penumbra ${ }^{18}$, que se puede beneficiar de la trombólisis intraarterial. Cuando la oclusión arterial ocurre en el territorio vertebrobasilar, se aceptan ventanas terapéuticas superiores a $3 \mathrm{~h}^{19}$. La oclusión de la arteria basilar implica un pésimo pronóstico $y$, tradicionalmente se ha considerado como una indicación de abordaje intraarterial incluso durante las primeras $24 \mathrm{~h}^{20,21}$. La asociación de un rápido tratamiento intravenoso seguido posteriormente de trombólisis intraarterial y reperfusión endovascular mecánica puede ser una alternativa si persiste la oclusión. El estudio Interventional Management of Stroke ${ }^{22}$ (fases 1 y 2) analizó la eficacia y la seguridad de la combinación del rt-PA intravenoso en dosis de $0,6 \mathrm{mg} / \mathrm{kg}$ seguido de tratamiento intraarterial hasta con una dosis máxima de $22 \mathrm{mg}$ en las 3 primeras horas del ictus isquémico. Las tasas de transformación hemorrágica fueron cercanas a las descritas en el estudio NINDS y aumentó el número de pacientes con mejor grado funcional a los 3 meses.

La eficacia y la seguridad del tratamiento trombolítico exclusivamente intraarterial se han constatado en diversos trabajos. El ensayo PROACT $\|^{23}$ evaluó el tratamiento intraarterial con prouroquinasas frente a placebo en el ictus por oclusión de la ACM dentro de las primeras $6 \mathrm{~h}$. En el grupo de tratamiento, las tasas de hemorragia intracraneal sintomática fueron del $10 \%$, con una recanalización del $66 \%$ (el $18 \%$ en el grupo control), a pesar de que el tiempo medio hasta el tratamiento fue de $5 \mathrm{~h}$ y media. A los 90 días, el $40 \%$ de los pacientes tratados con prouroquinasa tenía una escala de Rankin inferior o igual a 2 frente a un 25\% del grupo control, lo que demuestra la eficacia del tratamiento en términos de mejoría funcional. Un estudio multicéntrico japonés $(\mathrm{J}-\mathrm{MUSIC})^{24}$ refirió también buenos resultados funcionales en el grupo tratado con uroquinasa intraarterial (51\%) frente al grupo control (34\%; $p=0,01)$. El ensayo MELT valoró el tratamiento con bolos de uroquinasa intraarterial hasta la disolución del trombo más la opción de tratamiento mecánico con microcatéter. La ventana terapéutica fue de $6 \mathrm{~h}$, la tasa de recanalización del 74\% y la de hemorragia intracraneal sintomática del $9 \%{ }^{25}$. En nuestra serie, la tasa de recanalización fue del $68,7 \%$ y la de hemorragia intracraneal sintomática del 6,2\%, similares a las descritas en la literatura médica. En los últimos años, se está trasladando a las arterias intracraneales el uso con catéteres utilizados para la extracción y la disrupción mecánica de trombos en las arterias coronarias. Estos dispositivos rediseñados y adaptados a la circulación cerebral se han utilizado en los estudios $\mathrm{MERCl}$ y multi-MERCl ${ }^{26,27}$ en pacientes fuera de la ventana terapéutica $(<8 \mathrm{~h})$ o con criterios de exclusión para el tratamiento intravenoso. En 


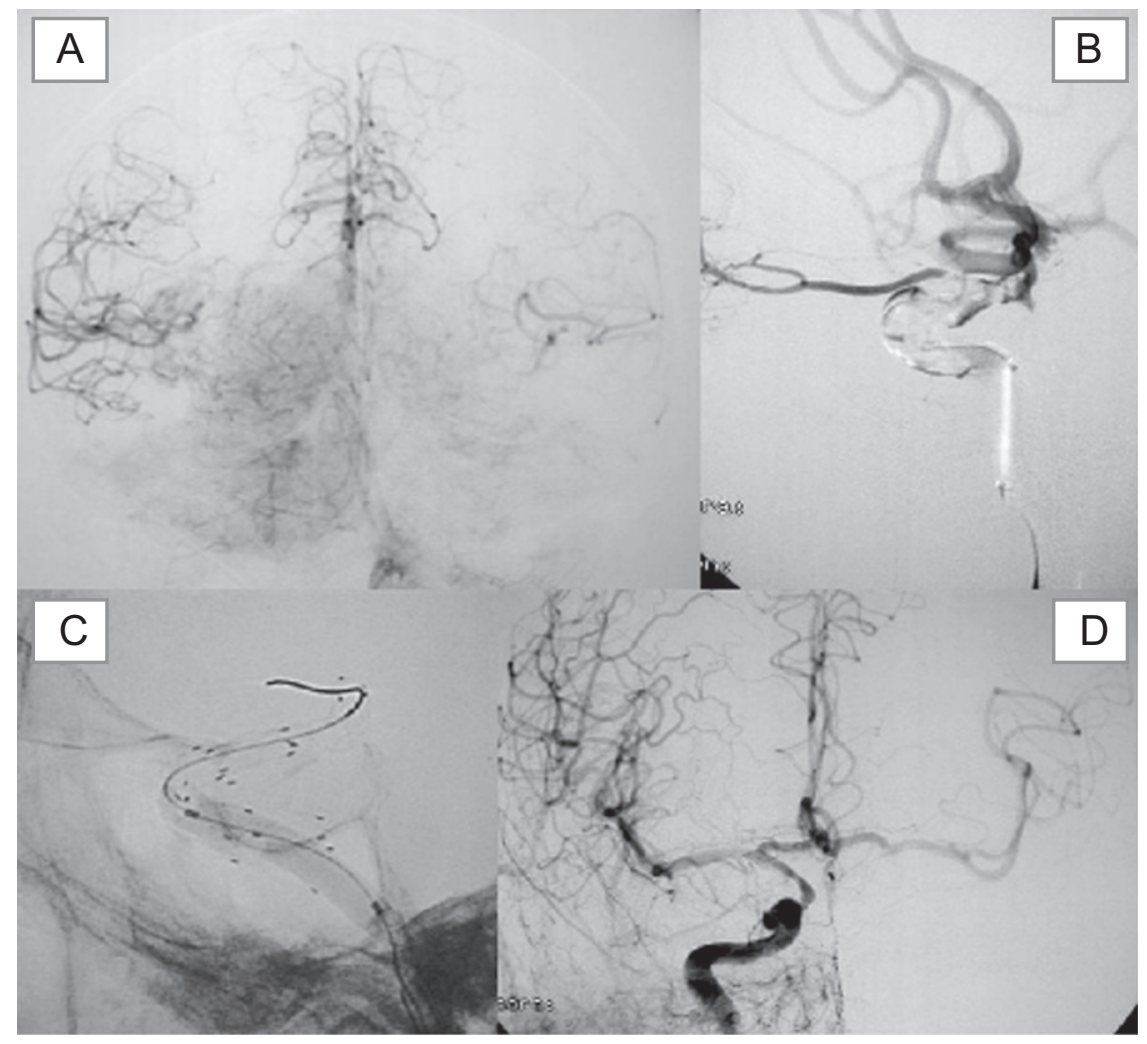

Figura 4 A) Arteriografía selectiva de la arteria vertebral a través de la que se rellena de forma muy tardía la circulación anterior. B) inyección selectiva carotídea derecha que muestra la gran carga de trombo intraluminal. C) Varios stents con balón de angioplastia hinchado como tratamiento mecánico del trombo. D) Resultado final con normalización de la circulación anterior que se rellena de forma anterógrada desde la arteria carótida derecha.

A

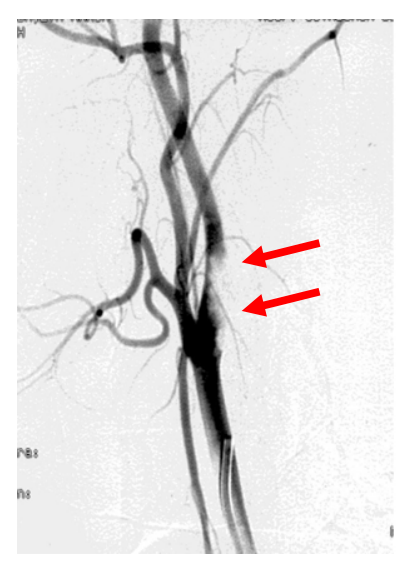

B

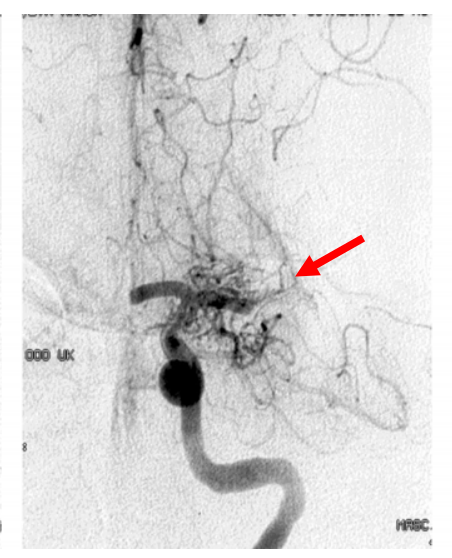

C

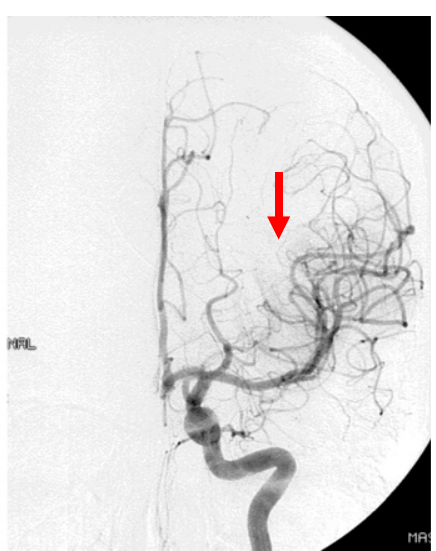

Figura 5 A) Arteriografía selectiva de la arteria carótida común derecha con gran defecto de repleción en el origen de la arteria carótida interna, que se corresponde con un trombo. B) Obstrucción asociada en el segmento M1 intracraneal de la arteria cerebral media. C) Control final tras la aspiración del trombo y la trombólisis farmacológica con 550.000 UI de uroquinasa.

ambos estudios se consiguieron altas tasas de recanalización (el 55 y el 68\% asociado a trombólisis intraarterial) con riesgo de hemorragia del $8-9 \%$. Cuatro de nuestros pacientes $(25 \%)$ requirieron embolectomía mecánica. Otra práctica habitual son las técnicas de angioplastia e implantación de stents. En nuestra serie se implantaron stents en 10 casos (62,5\%).

En la actualidad, se acepta sin discusión como primera opción el tratamiento con rt-PA intravenoso, pero sus estrictos criterios de exclusión, la limitada ventana terapéutica de $3 \mathrm{~h}$ y los pobres resultados en casos de obstrucción de gran vaso hacen que el tratamiento trombolítico intraarterial asociado o no al tratamiento intravenoso pueda ser una gran alternativa como se ha demostrado en varios estudios ${ }^{10-15,28-31}$. En nuestra serie, se obtuvieron unos buenos resultados funcionales con escasas complicaciones técnicas. Los 3 pacientes que evolucionaron a muerte encefálica presentaban gran 
Tabla 3 Indications for the Performance of Intracranial Endovascular Neurointerventional Procedures A Scientific Statement From the American Heart Association Council on Cardiovascular Radiology and Intervention, Stroke Council, Council on Cardiovascular Surgery and Anesthesia, Interdisciplinary Council on Peripheral Vascular Disease, and Interdisciplinary Council on Quality of Care and Outcomes Research ${ }^{33}$

Recomendaciones clase I:

1. «La trombólisis intraarterial es una opción de tratamiento para pacientes seleccionados con $<6 \mathrm{~h}$ de evolución y oclusión de la arteria cerebral media que no son candidatos al rt-PA intravenoso» (clase I, nivel de evidencia B)

2. «Este tratamiento exige la atención del paciente en un centro con posibilidades de acceso inmediato a una angiografía cerebral y con neurointervencionistas cualificados» (clase I, nivel de evidencia C)

3. «La disponibilidad de la trombólisis intraarterial nunca debería excluir de la trombólisis intravenosa a los pacientes candidatos» (clase I, nivel de evidencia A)

Recomendaciones clase II:

1. «En pacientes con contraindicaciones a la trombólisis intravenosa, tales como intervenciones quirúrgicas recientes, la trombólisis intraarterial es una opción razonable» (clase Ila, nivel de evidencia $\mathrm{C}$ )

rt-PA: activador tisular del plasminógeno recombinante.

extensión y gravedad del infarto con escalas de NIHSS al ingreso de 20-25, lo que justificaría esta evolución.

Las últimas Guidelines for the Early Management of Adults with Ischemic Stroke ${ }^{32}$ y las indicaciones para los procedimientos endovasculares intracraneales de la AHA del año $2009^{33}$ clasifican el tratamiento trombolítico intraarterial dentro de las 6 primeras horas en pacientes no candidatos al tratamiento intravenoso como recomendación clase ।, nivel de evidencia B (tabla 3). Una de las ventajas del tratamiento intraarterial es que, al poder administrar in situ una alta concentración del fármaco, se optimizan sus resultados, se requirieren dosis menores $\mathrm{y}$, por tanto, se minimizan sus efectos secundarios ${ }^{34,35}$. El problema del tratamiento intraarterial es que requiere una importante infraestructura con personal médico altamente entrenado y especializado y su mayor limitación es la escasa disponibilidad de un servicio de neuroradiología intervencionista de guardia. Actualmente, existe una cierta discriminación negativa en el tratamiento del ictus agudo, con clara desventaja en infraestructura y recursos humanos cuando se lo compara con la enfermedad coronaria, lo que se confirma en algunos estudios que demuestran que el tratamiento trombolítico intravenoso e intraarterial se utiliza, respectivamente, solamente en el 1,4 y el $0,3 \%$ de los pacientes ${ }^{36}$. Considerando que la enfermedad cerebrovascular constituye una de las primeras causas de mortalidad y discapacidad, su tratamiento multidisciplinario constituye, sin duda, uno de los desafíos más importantes para la sanidad en los próximos años ${ }^{37,38}$. En este sentido, la implantación de equipos neurointervencionistas de guardia debe ser motivo para una fuerte reivindicación a las administraciones correspondientes. El alto precio en mortalidad y calidad de vida de nuestros pacientes lo convierten en una inexcusable obligación ética y profesional.

\section{Bibliografía}

1. Navarrete P, Pina F, Rodríguez R, Murillo F, Jiménez D. Manejo inicial del ictus isquémico agudo. Med Intensiva. 2008;32: 431-43.

2. Herrera M, Gállego J, Muñoz R, Aymerich N, Zandio B. Reperfusión en el ictus isquémico agudo: estado actual y futuro. Anales Sis San Navarra. 2008;31:31-46.

3. Pérez Sempere A. Morbilidad por enfermedad cerebrovascular en España: incidencia y prevalencia. Rev Neurol. 1999;29: 879-81.

4. The National Institute of Neurological Disorders and Stroke rt-PA Stroke Study Group. Tissue plasminogen activator for acute ischaemic stroke. N Engl J Med. 1995;333:1581-7.

5. Wahlgren N, Ahmed N, Davalos A, Ford GA, Grond M, Hacke W, et al. Trombolysis with alteplase for acute ischaemic stroke the Safe Implementation of Thrombolysis in Stroke-Monitoring study (SITS-MOST): An observational study. Lancet. 2007;369:275-82.

6. Navarrete-Navarro P, García Alcántara A, Murillo Cabezas F, Vazquez Mata G, Fernández Fernández S, Jiménez Moragas JL, et al. La patología cerebrovascular aguda en las áreas de Cuidados Críticos de Andalucía. Análisis clínico-epidemiológicos y de práctica médica. Proyecto Evascan Med Intensiva. 2000;24:257-63.

7. Ribo M, Molina CA, Rovira A, Quintana M, Delgado P, Montaner J, et al. Safety and afficacy of intravenous tissue plasminógeno activator stroke treatment in the 3-to 6-hour window using multimodal transcranial Doppler/MRI selection protocol. Stroke. 2005;36:602-6.

8. Wahlgren N, Ahmed N, Dávalos A, Hacke W, Millán M, Muir K, et al. for the SITS investigators. Trombolysis with alteplase $3-4.5 \mathrm{~h}$ after ischaemic stroke (SITS-ISTR): An observational study. The Lancet. 2008;372:1303-9.

9. Thomalla G, Schwark C, Sobesky J, Bluhmki E, Fiebach JB, Fiehler J, et al. for the MRI in Acute Stroke Study Group of the German Competence Network Stroke. Outcome and symptomatic bleeding complications of intravenous thrombolysis within $6 \mathrm{~h}$ in MRI-selected stroke patients: Comparison of a German multicenter study with the pooled data of ATLANTIS, ECASS and NINDS tPA trials. Stroke. 2006;37:852-8.

10. Mangiafico S, Cellerini M, Nencini P, Gensini G, Inzitari D. Intravenous tirofiban with intra-arterial urokinase and mechanical thrombolysis in stroke: Preliminary experience in 11 cases. Stroke. 2005;36:2154-8.

11. Wolfe T, Suárez JI, Tarr RW, Welter E, Landis D, Sunshine JL. Comparision of combined venous and arterial thrombolysis with primary arterial therapy using recombinant tissue plasminogen activator in acute ischemic stroke. J Stroke Cerebrovasc Dis. 2008; 17:121-8.

12. Lewandowski CA, Frankel M, Tomsick TA, Broderick J, Frey J, Clark W, et al. Combined intravenous and intra-arterial r-TPA versus intra-arterial therapy of acute ischemic stroke: Emergency Management of Stroke (EMS) Bridging Trial. Stroke. 1999;30:2598-605.

13. Arnold M, Schroth G, Nedeltchev K, Loher T, Remonda L, Stepper F, et al. Intra-arterial thrombolysis in 100 patients with acute stroke due to middle cerebral artery occlusion. Stroke. 2002;33:1828-33.

14. Mattle HP, Arnold M, Georgiadis D, Baumann C, Nedeltchev K, Benninger D, et al. Comparison of intraarterial AND intravenous thrombolysis for ischemic stroke with hyperdense middle cerebral artery sign. Stroke. 2008;39:379-83.

15. Agarwal P, Kumar S, Hariharan S, Eshkar N, Verro P, Cohen B, et al. Hyperdense middle cerebral artery sign: Can it be used to select 
intra-arterial versus intravenous thrombolysis in acute ischemic stroke? Cerebrovasc Dis. 2004;17:182-90.

16. Saposnik G, Young B, Silver B, di Legge S, Webster F, Beletsky V, et al. Lack of improvement in patients with acute stroke after treatment with thrombolytic therapy: Predictors and association with outcome. JAMA. 2004;292:1839-44.

17. Kent DM, Selker HP, Ruthazer R, Bluhmki E, Hacke W. The stroke-thrombolytic predictive instrument: A predictive instrument for intravenous thrombolysis in acute ischemic stroke. Stroke. 2006;37:2957-62.

18. Darby DG, Barber PA, Gerraty RP, Desmond PM, Yang Q, Parson M, et al. Patophysiological topography of acute ischemia by combined diffusion-weighted and perfusion MRI. Stroke. 1999;30:2043-52.

19. Lindsberg PJ, Soinne L, Tatlisumak T, Roine RO, Kallela M, Happola 0 , et al. Long-term outcome after intravenous thrombolysis of basilar artery occlusion. JAMA. 2004;292:1862-6.

20. Lindsberg PJ, Mattle HP. Therapy of basilar artery occlusion: A systematic analysis comparing intra-arterial and intravenous thrombolysis. Stroke. 2006;37:922-8.

21. Macleod MR, Davis SM, Mitchell PJ, Gerraty RP, Fitt G, Hankey GJ, et al. Results of a multicentre, randomised controlled trial of intra-arterial urokinase in the treatment of acute posterior circulation ischaemic stroke. Cerebrovasc Dis. 2005;20:12-7.

22. Interventional Management of Stroke Investigators. The Interventional Management of Stroke (IMS) II Study. Stroke. 2007;38:2127-35.

23. Furlan A, Higashida R, Wechsler L, Gent M, Rowley H, Kase C, et al. Intra-arterial prourokinase for acute ischemic stroke. The PROACT II study: A randomized controlled trial. Prolyse in acute cerebral thromboembolism. JAMA. 1999;282:2003-11.

24. Inoue T, Kimura K, Minematsu K, Yamaguchi T, Japan Multicenter Stroke Investigator's Collaboration. A case-control analysis of intra-arterial urokinase thrombolysis in acute cardioembolic stroke. Cerebrovasc Dis. 2005;19:225-8.

25. Ogawa A, Mori E, Minematsu K, Taki W, Takahashi A, Nemoto S, et al. for The MELT Japan Study Group. Randomized trial of intraarterial infusion of urokinase within $6 \mathrm{~h}$ of middle cerebral artery stroke: The Middle cerebral artery Embolism Local fibrinolytic intervention Trial (MELT) Japan. Stroke. 2007;38:2633-9.

26. Smith WS, Sung G, Starkman S, et al. Safety and efficacy of mechanical embolectomy in acute ischemic stroke: Results of the MERCI trial. Stroke. 2005;36:1432-8.

27. Smith WS. Safety of mechanical thrombectomy and intravenous tissue plasminogen activator in acute ischemic stroke. Results of the multi mechanical embolus removal in cerebral ischemia (MERCI) trial, part I. AJNR Am J Neuroradiol. 2006;27:1177-82.
28. IMS Study Investigators. Combined intravenous and intraarterial recanalization for acute ischemic stroke: The Interventional Management of Stroke Study. Stroke. 2004;35:904-11.

29. Choi JB, Bateman BT, Mangla S, Marshall RS, Prabhakaran S, Chong J, et al. Endovascular recanalization therapy in acute isquemic stroke. Stroke. 2006;37:419-24.

30. Lisboa RC, Jovanovic BD, Alberts MJ. Analysis of the safety and efficacy of intra-arterial thrombolytic therapy in ischemic stroke. Stroke. 2002;33:2866-71.

31. Sen S, Huang DY, Akhavan O, Wilson S, Verro P, Solander S. IV vs. IATPA in acute ischemic stroke with CT angiographic evidence of major vessel Occlusion: A feasibility study. Neurocrit Care. 2009;11:76-81.

32. Adams H, del Zoppo G, Alberts M, Bhatt DL, Brass L, Furlan A, et al. Guidelines for the early management of adults with ischemic stroke. A guideline from the American Heart Association/ American Stroke Association Stroke Council, Clinical Cardiology Council, Cardiovascular Radiology and Intervention Council, and the Atherosclerotic Peripheral Vascular Disease and Quality of Care Outcomes in Research Interdisciplinary Working Groups. The American Academy of Neurology affirms the value of this guideline as an educational tool for neurologists. Circulation. 2007;115:e478-534.

33. Meyers PM, Schumacher HC, Higashida RT, Barnwell SL, Creager MA, Gupta R, et al. Indications for the Performance of Intracranial Endovascular Neurointerventional Procedures A Scientific Statement From the American Heart Association Council on Cardiovascular Radiology and Intervention, Stroke Council, Council on Cardiovascular Surgery and Anesthesia, Interdisciplinary Council on Peripheral Vascular Disease, and Interdisciplinary Council on Quality of Care and Outcomes Research. Circulation. 2009;28:2235-49.

34. Janjua N, Brisman JL. Endovascular treatment of acute ischaemic stroke. Lancet Neurol. 2007;6:1086-93.

35. Qureshi Al. Endovascular treatment of cerebrovascular diseases and intracranial neoplasms. Lancet. 2004;363:804-13.

36. Qureshi Al, Kirmani JF, Sayed MA, Siddiqui AM, Safdar A, Pande RU, et al. Buffalo metropolitan area and Erie County stroke study: Rationale, design, and methods. Neuroepidemiology. 2004;23: 289-98.

37. Camacho JA, Jurado B, Jiménez JM, Montijano AJ, de Molina A. Ictus y trombolisis en un hospital básico. Un deber posible. Med Intensiva. 2006;30:238-9.

38. Roca Guiseris J, Pérez-Villaves JM, Navarrete-Navarro P. Accidente cerebrovascular agudo. ¿Ampliamos nuestra cartera de servicios?Med Intensiva 2003;27:596-7. 